\title{
Characterization of a Mechanically Tunable Gyroid Photonic Crystal Inspired by the Butterfly Parides Sesostris
}

\section{Citation}

Pouya, Caroline, Johannes T. B. Overvelde, Mathias Kolle, Joanna Aizenberg, Katia Bertoldi, James C. Weaver, and Pete Vukusic. 2015. "Characterization of a Mechanically Tunable Gyroid Photonic Crystal Inspired by the Butterfly Parides Sesostris." Advanced Optical Materials 4 (1) (September 25): 99-105. doi:10.1002/adom.201500436.

\section{Published Version}

doi:10.1002/adom.201500436

\section{Permanent link}

http://nrs.harvard.edu/urn-3:HUL.InstRepos:37254717

\section{Terms of Use}

This article was downloaded from Harvard University's DASH repository, and is made available under the terms and conditions applicable to Open Access Policy Articles, as set forth at http:// nrs.harvard.edu/urn-3:HUL.InstRepos:dash.current.terms-of-use\#OAP

\section{Share Your Story}

The Harvard community has made this article openly available.

Please share how this access benefits you. Submit a story.

Accessibility 


\title{
WILEY-VCH
}

DOI: $10.1002 /(($ please add manuscript number $))$

Article type: Communication

\section{Characterization of a Mechanically Tunable Gyroid Photonic Crystal Inspired by the Butterfly Parides sesostris}

Caroline Pouya*, Johannes T. B. Overvelde, Mathias Kolle, Joanna Aizenberg, Katia Bertoldi, James C. Weaver, and Pete Vukusic*

Dr C. Pouya, Prof. P. Vukusic

School of Physics and Astronomy, University of Exeter, Exeter, EX4 4QL, United Kingdom E-mail: C.Pouya@exeter.ac.uk, P.Vukusic@exeter.ac.uk

J. T. B. Overvelde, Prof. K. Bertoldi

School of Engineering and Applied Sciences, Harvard University, Cambridge, MA, 02138, USA

Dr M. Kolle, Prof. J. Aizenberg, Dr J. C. Weaver

Wyss Institute for Biologically Inspired Engineering, Harvard University, Cambridge, MA, 02138, USA

Dr M. Kolle,

Department of Mechanical Engineering, MIT, Cambridge, MA, 02139, USA

Keywords: (photonic crystal, butterfly, gyroid, mechanical stress, mechanical tuning)

\begin{abstract}
:
Dynamically tunable three-dimensional photonic materials offer potential for the development of novel optical technologies. For spectral ranges of interest, however, the systematic and high-throughput characterization of their optical responses as a function of physical deformation remains elusive. This arises from challenges associated with their nanoscale fabrication, deformation, and structural and optical characterization. By exploiting the scale-invariance of Maxwell's equations and of the elastic material response, we demonstrate the systematic characterization of a tunable macro-scale replica of the gyroid structure found in Parides sesostris butterflies' wing scales. This scaled replica of the natural photonic crystal was fabricated from a UV cross-linkable elastomer capable of large-range reversible linear deformation via high-resolution additive manufacturing. The controlled
\end{abstract}




\section{WILEY-VCH}

deformation of this periodic elastomer-based photonic crystal allowed for a systematic variation of its optical response. Microwave spectroscopy, electromagnetic and mechanical Finite-Element Analysis, and x-ray computed micro-tomography were used to gain a detailed understanding of the gyroid's compression-induced deformation and the resulting modification of its electromagnetic properties. This approach enables controlled and rapid prototyping and optimization of any desired photonic architecture at macroscopic dimensions prior to the fabrication of its nano/microscopic analogue relevant for manipulating smaller wavelength radiation for use in a wide range of technological applications.

\section{Introduction}

Photonic crystals ${ }^{[1-5]}$ (PCs) can be used to selectively reflect specific frequencies of electromagnetic radiation. Since most PCs are static in material composition and geometry, their reflection or transmission behavior can only be changed through angular reorientation. In contrast, flexible PCs can exhibit tunable photonic responses, ${ }^{[3,5-7]}$ where reflected or transmitted frequencies can be controlled through structural deformation. Tunable responses can also be achieved via the application of voltage, ${ }^{[8-9]}$ by adsorption of vapors, ${ }^{[10-11]}$ via temperature modulation, ${ }^{[12-13]}$ or by the application of strain. ${ }^{[14-16]}$ Recently, increased efforts have been aimed at fabricating one- ${ }^{[16]}$ two- ${ }^{[17-18]}$, and three-dimensional ${ }^{[19]}$ tunable PCs. Since the requirements imposed on PC geometry and material compositions vary between applications, the development of robust fabrication strategies for the customizable and largescale production of 3D PCs is a research area of great interest. Accordingly, material scientists have embraced the study of structurally colored biological photonic systems to gain inspiration for synthesis routes that can facilitate the fabrication of novel optical materials with exploitable properties. ${ }^{[16-20]}$ Such biological photonic structures found in terrestrial and aquatic environments typically comprise polymeric materials including keratin, cellulose, and chitin. They exhibit a wide range of geometries including colloidal particles, ${ }^{[21-22]}$ 


\section{WILEY-VCH}

continuous $^{[23]}$ or discrete units of multilayers, ${ }^{[24-25]}$ concentric cylinders ${ }^{[16,26]}$, and bicontinuous gyroids. ${ }^{[27-30]}$ Despite the promise of new biologically-inspired design routes for photonic material production, there is currently a dearth of cost-effective high-throughput methods by which synthetic analogues of these biophotonic materials can be fabricated.

In an effort to address these limitations, we have utilized an efficient experimental approach to investigate electromagnetic responses ${ }^{[31-32]}$ and structural properties of tunable gyroid geometries. Using the naturally occurring photonic gyroid found in the wing scales of Parides sesostris butterflies ${ }^{[28]}$ as an experimental model system (Figure 1), we employed high-resolution additive manufacturing to replicate its 3D architectural details in a reversibly deformable macro-scale analogue. We specifically used a filling fraction of $40 \%$ that closely matches the filling fraction of the butterfly gyroid. ${ }^{[28]}$. The tunability of the deformable PC's electromagnetic properties can be investigated by imposing controlled anisotropic deformations of the crystal structure through compressive loading. By fabricating flexible dielectric PCs with centimeter-scale unit cells, it is possible to produce materials with tunable photonic responses in the microwave regime $(0.3 \mathrm{GHz}-300 \mathrm{GHz})$. By exploiting the scaleinvariance of Maxwell's equations and of the constituent laws of solid mechanics, the structure-function relationships of photonic materials can be directly investigated. By working at the macro-scale, we are taking full advantage of the design freedom of additive manufacturing, comprehensive optical characterization using microwave technology, and the in-situ 3D structural characterization capabilities of micro-scale x-ray computed tomography. The complex gyroid morphology was chosen as a model system to demonstrate the power of this approach for gaining systematic and comprehensive insight into the behavior of macroscale photonic materials. Our method could readily be applied to assess any other structural motif in a similar manner. Once the fundamental structure-function relationships underlying the dynamics of reversibly deformable photonic materials are understood at the macro-scale, this insight can be applied for the realization of structural motifs at smaller length scales to 


\section{WILEY-VCH}

create tunable photonic devices for the Terahertz, Telecom, Near-IR, visible, UV, and x-ray frequency ranges.

\section{Results and discussion}

Inspection of $P$. sesostris (Figure 1) reveal that the brightly colored metallic appearance of the green wing patches originates from scales that are not individually resolvable by the human eye (Figure 1C-E). These scales have a complex morphology, including surface ridges (Figure $1 \mathrm{D}-\mathrm{G})^{[33-35]}$, spectrally-selective pigment-based absorbers, as proposed by Wilts et al., ${ }^{[35]}$ and an underlying gyroid photonic crystal (Figure $\left.1 \mathrm{G}\right)^{[28,33-38] \text {, }}$

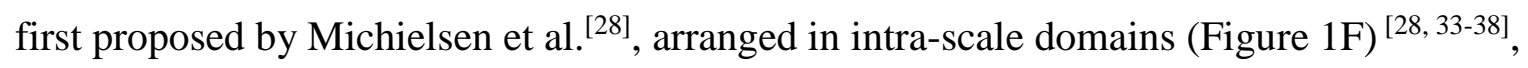
which are all expected to contribute to the scales' optical appearance. ${ }^{[28,30,35-38]}$ A graphical representation of the gyroid is presented in Figure 1H, with its unit cell highlighted in green. Using the key dimensional parameters obtained from previous transmission electron microscopy (TEM) studies, ${ }^{[28]}$ a flexible macro-scale model of the gyroid was fabricated using high-resolution 3D printing (Figure 2A-B). The resulting structure was investigated to see if its photonic response could be deliberately tuned and controlled by systematically varying the applied compressive load.

In order to characterize the gyroid's deformation modes, x-ray computed microtomographic (microCT) reconstructions were obtained from the flexible 3D-printed models at various stages of compression (Figure 3). The reconstructions of the deformed gyroid models were then compared to the results obtained from the finite element analysis (FEA) compression simulations (Figure 4A). The non-linear finite element simulations were performed on virtual gyroid models constructed from approximately 10,000 tetrahedral elements per unit cell, which were compressed uniaxially by applying a vertical displacement to the top face, while leaving all lateral faces free to expand. The results obtained from these 


\section{WILEY-VCH}

studies revealed that the mechanical response of the gyroid was only moderately affected by the number of unit cells. Models comprising $3 \times 3 \times 3,5 \times 5 \times 5$, and $7 \times 7 \times 7$ unit cells exhibited a similar extent of lateral expansion. In particular, for a gyroid containing $7 \times 7 \times 7$ unit cells, the maximum lateral expansion was measured to be $2.15 \%, 4.38 \%, 6.78 \%$, and $9.25 \%$ for applied strain values of $5.6 \%, 11.1 \%, 16.7 \%$, and $22.2 \%$, respectively. These values correlated well with the $10 \times 10 \times 10$ structure tested experimentally, in which the maximum lateral expansion was observed to be $9.7 \%$ at a compression of $22.2 \%$. The results of these simulations are presented in Figure 4, clearly showing the large geometric changes induced by the applied

compression. The contour colors correspond to the Von Mises stress $\left(\sigma_{V M}=\sqrt{\frac{3}{2} \sigma_{i j} \sigma_{i j}-\frac{1}{2} \sigma_{k k}^{2}}\right.$, $\sigma_{i j}$ denoting a component of the Cauchy stress), where the most highly stressed regions are denoted in red. Significantly, these results revealed that, at moderate compressions, the stress is very uniformly distributed and does not tend to localize, a property that would dramatically improve the system's toughness. This result could be explained based on the gyroid's highly smooth minimal surface geometry.

Excluding the boundary effects where the physical gyroid model contacted the compression plates, the x-ray computed micro-tomography and FEA data sets agreed surprisingly well within the bulk core of the gyroid, thereby providing a robust model for performing realistic microwave electromagnetic transmission simulations. Figures $4 \mathrm{~B}$ and $4 \mathrm{C}$ show the theoretical and experimental transmitted field magnitude data, respectively, obtained using transverse magnetic incident polarization, with respect to the plane of incident radiation, from the compliant gyroid. The structure was compressed in the [100] direction by $0 \%, 5.6 \%$, $11.1 \%, 16.7 \%$, and $22.2 \%$ of the original size of the gyroid model. The observed frequency shifts that occurred upon compression of the sample were similar for both transverse magnetic and the equivalent transverse electric polarizations. Based on these observations and since the linear polarization dependence of the gyroid has been discussed previously, ${ }^{[29,}{ }^{32]}$ the 


\section{WILEY-VCH}

transverse electric results are not presented here. The sample was positioned at an azimuthal angle of $\varphi=0^{\circ}$ and was rotated over a polar angle range of $-45^{\circ} \leq \theta \leq 45^{\circ}$ for each compressed state (Figure 2C). The dark bands in Figure 4B and 4C are representative of low electromagnetic transmission through the gyroid photonic crystal structure for each of the indicated states of compression. The frequency axes are presented in normalized reduced frequency units of $c / a$ where $c$ is the speed of light in free space and $a$ is the uncompressed lattice constant of $9 \mathrm{~mm}$. In the native, uncompressed state, two of the transmission minima (labelled ' $\mathrm{P}$ ' and 'Q' on the central graph of Figure 4B) are in very close proximity and appear to overlap or merge, particularly at normal incidence. As compression of the sample begins to extend beyond $11.1 \%$, these previously overlapping features separate into two discrete and distinctly resolvable bands ('P' and 'Q'). From theoretical electric field plots (Figure S1, Supporting Information) and geometric analyses, these two features originate

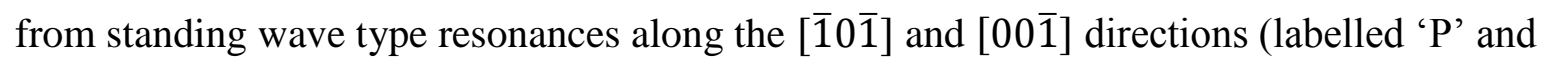
'Q', respectively, on the central graph of Figure 4B). The resonant feature labelled 'P' on the central graph of Figure 4B also results from significant absorption. When the structure is uncompressed, the frequency position of these features also lies close to the crossing point of a third feature (labelled ' $R$ ' on Figure 4B) which originates from a standing wave type resonance along the $[0 \overline{1} \overline{1}]$ direction. When compressed by $22.2 \%$, in the [100] direction as described, the gyroid model's central region expands by $9.7 \%$ along the $[00 \overline{1}]$ direction. From trigonometric analysis, and by using these expansion values, we can identify similar

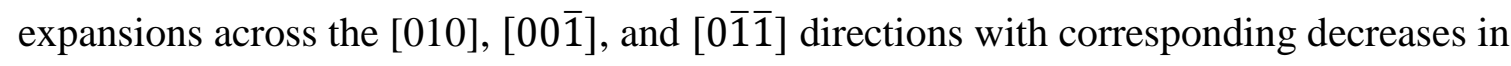
frequency of the resonant features (' $Q$ ' and ' $R$ ' on Figure 4B) associated with these directions. Similarly, the structural configurations in the [100] and [ $[\overline{1} 0 \overline{1}]$ directions are reduced to $77.8 \%$ and $95.1 \%$ of their original sizes: Figure $4 \mathrm{~B}$ and $4 \mathrm{C}$ show a corresponding increase in frequency of the associated resonant features along these directions (labelled 'P' on Figure 


\section{WILEY-VCH}

4B). At $22.2 \%$ compression, the experimentally determined frequency shifts of each transmission minimum, in reduced units, were measured to be 0.072 (c/a), 0.039 (c/a), and 0.177 (c/a) (Figure 4C) for the features labelled 'R', 'Q', and 'P', respectively, in Figure 4B. These values were measured at the polar angle of $45^{\circ}$ (Figure 4C). As a result of the gyroid's bicontinuous nature (consisting of a solid/polymer phase and a void/air phase), the application of a compressive load results in a net volume reduction of the structure (Figure 3). This increase in material filling fraction directly corresponds to a slight narrowing of the transmission minima, which is observed both theoretically (Figure 4B) and experimentally (Figure 4C). A very slight red-shift in frequency is also expected from a change in filling fraction away from the optimum condition for large stop-band width as occurs in the uncompressed gyroid structure. ${ }^{[28,32]}$ For the simulated gyroid modelling, when the maximum compression of $22.2 \%$ was applied to the structure, the frequency shifts of each of the corresponding features labelled 'R', 'Q', and 'P', respectively, in the theoretical data plots (Figure 4B) were measured to be approximately $0.057,0.033$, and 0.165 , in reduced frequency units $(c / a)$. These values were also measured at the polar angle of $45^{\circ}$ (Figure $4 \mathrm{C}$ ). These frequency changes were marginally smaller than those obtained from the experimental measurements, with a slight variability in filling fraction and the lack of graded expansions, being the most likely contributing factors. ${ }^{[39]}$

In conclusion, through both theoretical and experimental approaches, in this study, we have performed a quantitative assessment of a flexible gyroid photonic crystal structure and compression-dependant optical response. We found that, when compressed, previously overlapping transmission minima spectrally separate. While this observed overlap in dispersion features and consequent shifting behavior will only occur for specific geometries, similar results are predicted for other photonic crystal structures that exhibit a similar bcc Bravais lattice symmetry. Through the structural investigation, we also found that the gyroid does not tend to localize stresses but uniformly distributes them at moderate compressions. 


\section{WILEY-VCH}

This structural property is of particular importance from a mechanical engineering perspective as it would greatly enhance the toughness of the system. A robust sample that can withstand variable degrees of structural deformation is a necessity when designing and mechanically tuning a robust photonic system.

As shown in these studies, the capacity of 3D printing to rapidly create prototypes of almost any morphology in a broad range of material compositions makes it an ideal tool to build and systematically experimentally investigate macro-scale photonic materials that can be assessed electromagnetically in the microwave regime and structurally characterized through microCT. Taken holistically, the experimental approaches presented here highlight an efficient, high throughput, and cost-effective research platform for investigating the mechanisms by which the photonic response of periodic geometric solids can be dynamically tuned for a specific application of interest, while providing critical design cues for the fabrication of their micro- and nano-scale structural analogues.

\section{Experimental Section}

Research species: Dry specimens of Parides sesostris were acquired from commercial sources (Worldwide Butterflies (http://www.wwb.co.uk)). For optical characterization, the wings were imaged with a Keyence digital microscope (VHX-2000). The same samples were then mounted to conductive carbon tape, sputter-coated with gold and examined with a Tescan VegaIII scanning electron microscope (SEM). For the generation of electron beam Moiré patterns, the SEM scan line periodicity was matched to the ridge periodicity in the wing scales, thus permitting the illustration of nanoscale structural periodicity in a macroscale sample. Using information from previously published transmission electron microscopy studies, ${ }^{[28,34]}$ the critical parameters of the gyroid structure, principally its filling fraction of $40 \%$, were identified. 


\section{WILEY-VCH}

Gyroid modelling and fabrication: Gyroid model rendering for 3D-printing

compatible STereoLithography (.stl) files was achieved with a custom-made MATLAB code. ${ }^{[40]}$ Briefly, level set functions were used as versatile approximations to calculate intermaterial dividing surfaces of the gyroid. ${ }^{[28,41-45]}$ In particular, to describe the surface of the gyroid structure investigated in this study, we used Equation 1 with $\mathrm{t}=-0.3$ and $\mathrm{L}=9 \mathrm{~mm} .{ }^{[40]}$

$$
\sin \left(\frac{2 \pi}{L} x\right) \cos \left(\frac{2 \pi}{L} y\right)+\sin \left(\frac{2 \pi}{L} y\right) \cos \left(\frac{2 \pi}{L} z\right)+\sin \left(\frac{2 \pi}{L} z\right) \cos \left(\frac{2 \pi}{L} x\right)=t
$$

Vertices and facets of these surfaces were then rendered using MATLAB's isosurface function. Facet normals and vertex coordinates were written to an STL file directly from MATLAB using a custom script. The resulting 3D model had a material filling fraction of $40 \%$ and a lattice constant $a=9 \mathrm{~mm}$ (Figure 2-3), designed so that the model's structure was directly analogous to the $P$. sesostris wing-scale gyroid system ${ }^{[28]}$ but suitable for electromagnetic characterization using microwave radiation. The models were then fabricated using a Stratasys Connex500 3D printer from a rubber-like UV cross-linkable material (TangoBlack+). The model was fabricated using a $32 \mu \mathrm{m}$ z-step height which resulted in a high quality smooth surface finish without any visible layers (a common problem for thermoplastic filament-based 3D printing methods). The fabrication process lasted several hours. Based on established techniques, ${ }^{[46,47]}$ the material permittivity was measured to be $\varepsilon=$ $2.85+0.15 \mathrm{i}$ in the frequency regime used for our experiments. The key geometrical parameter that was replicated from the $P$. sesostris gyroid was the material filling fraction which was 40\%. ${ }^{[28]}$ The remaining volume was air, which was easily displaced throughout the system upon compression due to the bicontinuous nature of the gyroid. Gyroids were fabricated with $5 \times 5 \times 5$ and $10 \times 10 \times 10$ unit cells dimensions in order to explore the high- and low-resolution response of this material under different loading regimes. For the microwave studies, a 


\section{WILEY-VCH}

$10 \times 10 \times 10$ unit cells structure was fabricated such that the incident face was parallel to the (001) set of periodic planes of its photonic structure.

Micro-computed tomography studies: For microCT studies, the gyroid model was scaled up by a factor of two and the $3 \mathrm{D}$ model was reprinted in a $5 \times 5 \times 5$ geometry in order to obtain better $\mathrm{x}$-ray transmission and a more detailed 3D reconstruction during the various stages of deformation. In order to visualize the gyroid under compression at the specific strain levels of interest, we immobilized the specimen using a fixture made of acrylic plates, nylon bolts/ nuts and $2.5 \mathrm{~cm}$-thick rigid closed-cell foam plates placed between the specimen and the fixture (see Figure S2, Supporting Information). The foam plates were used as low-electron-density spacers that would be nearly invisible in the acquired x-ray transmission images and thus not interfere with volume rendering of the higher-electron-density gyroid. The gyroid/fixture assembly was then placed into a XRA-002 X-Tek microCT system for image data collection. The 3D reconstructions were performed using CT-Pro (Nikon Metrology) and the surface renderings were generated using VGStudio Max.

Gyroid modelling for finite element analysis compression studies: Static non-linear finite element analysis (FEA) of the gyroid structure was performed using the commercial finite element software Abaqus/Standard. The material was modelled using a nearly incompressible neo-Hookean model, whose energy is given by $W=\frac{\mu}{2}\left(\overline{I_{1}}-3\right)+\frac{K}{2}(J-1)^{2}$, where $\mu$ and $\mathrm{K}$ are the initial shear and bulk moduli, respectively. Moreover, $\mathrm{J}=\operatorname{det} \mathbf{F}$ and $\overline{I_{1}}=J^{-\frac{2}{3}}\left(\boldsymbol{F}^{\boldsymbol{T}} \boldsymbol{F}\right)$, where $\mathbf{F}$ is the deformation gradient. Note that in all our simulations we assumed that $\mathrm{K} / \mu=10000$, so that the material response is nearly incompressible. The mesh was built using linear hybrid solid elements (Abaqus element type C3D4H). Models comprising $3 \times 3 \times 3,5 \times 5 \times 5$, and $7 \times 7 \times 7$ unit cells were compressed uniaxially by applying a vertical displacement to the top face, while leaving all lateral faces free to expand. During the 


\section{WILEY-VCH}

analysis the maximum lateral expansion was monitored as a function of the applied compression. The results obtained from the FEA compression simulations agreed well with the compression results obtained from the experimental microCT studies and were thus used as a basis for the corresponding microwave simulations.

Experimental and simulated electromagnetic studies: For the experimental electromagnetic characterization, the compliant $10 \times 10 \times 10$ gyroid was placed inside a custom-designed compression device (Figure 2C), which was centered between aligned source and detector microwave broadband horns (Flann model DP241-AB; dual-polarized horn; option 10-50 $\mathrm{GHz}$ ). These were connected to an Anritsu Vector Star $70 \mathrm{kHz}$ to $70 \mathrm{GHz}$ vector network analyzer (VNA) (Figure 2C). The source horn emitted a Gaussian microwave beam with a full width half maximum of approximately $5 \mathrm{~cm}$ measured at a distance of $22 \mathrm{~cm}$ away from the source, which corresponded to the relative sample position in the experimental set-up. ${ }^{[39]}$ The compression device applied a force to the gyroid in a direction orthogonal to that of the incident beam as shown in Figure 2C. The gyroid was rotated over a polar angle range of $45^{\circ} \leq \theta \leq 45^{\circ}$ at each compressed state and the transmitted magnitude was recorded. Due to the number of unit cells across the sample and the signal attenuation by the constituent material, absorption occurred, particularly at the edges of resonant electromagnetic features and at higher frequencies. As a result, transmitted microwave magnitude was used in all graphical plots to clearly illustrate the presence and frequency change of the electromagnetic features. The sample was compressed by $0 \%, 5.6 \%, 11.1 \%, 16.7 \%$, and $22.2 \%$ of the initial array size and the resulting transmitted magnitude data was recorded.

In order to perform analogous numerical modelling of the transmission, the finite element method software HFSS was used (Ansoft HFSS, Version 13.0.0, ANSYS, Inc.). When building the model, the sample was assumed to expand uniformly in directions orthogonal to that of compression, an assumption which was validated by the FEA deformation analysis and experiments. The expansion value was taken as the measured 


\section{WILEY-VCH}

maximum expansion across the mid-plane of the structure since the Gaussian beam used in the experiments centers most of its power in this region. The electromagnetic simulation was performed on a gyroid array comprising 5 unit cells in the comparable $z$-direction, and infinite in the $x$ and $y$ directions for computing efficiency. The same real and imaginary values of permittivity were used to define material properties in the simulations. Using fewer unit cells along this z-direction results in a reduction of signal attenuation by the constituent materials, compared to the fabricated sample. An incident plane wave was used for excitation in the model and the transmitted electromagnetic field magnitude was measured over a range of angle and compression values. The initial lattice constant, filling fraction, and refractive indices used in the modelling were identical to those of the sample used in the electromagnetic experiments.

\section{Supporting Information}

Supporting Information is available from the Wiley Online Library or from the author.

\section{Acknowledgements}

The authors would like to thank Nick Cole for construction of the custom-made compression device used in the microwave spectroscopy experiments and Dr. Maik Scherer for providing a customized MATLAB code for the rendering of virtual gyroid models. C. Pouya acknowledges financial support from The University of Exeter EPSRC DTA. M. Kolle acknowledges financial support from the Alexander von Humboldt Foundation in the form of a Feodor-Lynen Postdoctoral Research Fellowship. This work was also supported by AFOSR Multidisciplinary University Research Initiative under FA9550-10-1-0020 and FA9550-09-10669-DOD35CAP. This work was performed in part at Harvard University's Center for Nanoscale Systems (CNS), a member of the National Nanotechnology Infrastructure Network (NNIN), which is supported by the National Science Foundation under NSF award no. ECS0335765 .

Received: ((will be filled in by the editorial staff))

Revised: ((will be filled in by the editorial staff)) Published online: ((will be filled in by the editorial staff))

[1] J. C. Knight, J. Broeng, T. A. Birks, P. S. J. Russell, Science (Washington, DC, U. S.)

1998, 282, 1476.

[2] P. Russell, Science (Washington, DC, U. S.) 2003, 299, 358. 


\section{WILEY-VCH}

[3] M. Muller, A. Bauer, T. Lehnhardt, A. Forchel, IEEE Photonics Technol. Lett. 2008, 20, 1100.

[4] H-W. Huang, C-H. Lin, Z-K. Huang, K-Y. Lee, C-C. Yu, H-C. Kuo, Jpn. J. Appl. Phys. 2010, 49, 022101.

[5] D. Sridharan, R. Bose, H. Kim, G. S. Solomon, E. Waks, Opt. Express 2011, 19, 5551.

[6] J. Xua, Z. Guo, J. Colloid Interface Sci. 2013, 406, 1.

[7] A. C. Arsenault, T. J. Clark, G. Von Freymann, L. Cademartiri, R. Sapienza, J. Bertolotti, E. Vekris, S. Wong, V. Kitaev, I. Manners, R. Z. Wang, S. John, D. Wiersma, G. A. Ozin, Nat. Mater. 2006, 5, 179.

[8] M. W. Haakestad, T. T. Alkeskjold, M. D. Nielsen, L. Scolari, J. Riishede, H. E. Engan, A. Bjarklev, IEEE Photonics Technol. Lett. 2005, 17, 819.

[9] A. C. Arsenault, D. P. Puzzo, I. Manners, G. A. Ozin, Nat. Photonics 2007, 1, 468.

[10] S. Mosor, J. Hendrickson, B. C. Richards, J. Sweet, G. Khitrova, H. M. Gibbs, T. Yoshie, A. Scherer, O. B. Shchekin, D. G. Deppe, Appl. Phys. Lett. 2005, 87, 141105.

[11] R. A. Potyrailo, T. A. Starkey, P. Vukusic, H. Ghiradella, M. Vasudev, T. Bunning, R. R.

Naik, Z. Tang, M. Larsen, T. Deng, S. Zhong, M. Palacios, J. C. Grande, G. Zorn, G. Goddard, S. Zalubovsky, Proc. Natl. Acad. Sci. U. S. A. 2013, 110, 15567.

[12] A. Faraon, J. Vuckovic, Appl. Phys. Lett. 2009, 95, 043102.

[13] J. Sussman, D. Snoswell, A. Kontogeorgos, J. J. Baumberg, P. Spahn, Appl. Phys. Lett. 2009, 95, 173116.

[14] W. Park, J. B. Lee, Appl. Phys. Lett. 2004, 85, 4845.

[15] M. Kolle, B. Zheng, N. Gibbons, J. J. Baumberg, U. Steiner, Opt. Express 2010, 18, 4356.

[16] M. Kolle, A. Lethbridge, M. Kreysing, J. J. Baumberg, J. Aizenberg, P. Vukusic, Adv. Mater. (Weinheim, Ger.) 2013, 25, 2239.

[17] B. Maune, M. Loncar, J. Witzens, M. Hochberg, T. Baehr-Jones, D. Psaltis, A. Scherer, Appl. Phys. Lett. 2004, 85, 360. 


\section{WILEY-VCH}

[18] A. Faraon, D. Englund, D. Bulla, B. Luther-Davies, B. J. Eggleton, N. Stoltz, P. Petroff, J. Vučković, Appl. Phys. Lett. 2008, 92, 043123.

[19] C. E. Finlayson, C. Goddard, E. Papachristodoulou, D. R. E. Snoswell, A. Kontogeorgos, P. Spahn, G. P. Hellmann, O. Hess, J. J. Baumberg, Opt. Express 2011, 19, 3144.

[20] H. Fudouzi, Sci. Technol. Adv. Mater. (Weinheim, Ger.) 2011, 12, 064704.

[21] S. M. Luke, B. T. Hallam, P. Vukusic, Appl. Opt. 2010, 49, 4246.

[22] P. Simonis, J. P. Vigneron, Phys. Rev. E: Stat., Nonlinear, Soft Matter Phys. 2011, 83, 011908.

[23] A. E. Seago, P. Brady, J. P. Vigneron, T. D. Schultz, J. R. Soc., Interface 2009, 6, S165.

[24] P. Vukusic, J. R. Sambles, C. R. Lawrence, R. J. Wootton, Proc. R. Soc. B 1999, 266, 1403.

[25] T. H. Chiou, L. M. Mäthger, R. T. Hanlon,T. W. Cronin, J. Exp. Biol. 2007, 210, 3624.

[26] T. M. Trzeciak, P. Vukusic, Phys. Rev. E: Stat., Nonlinear, Soft Matter Phys. 2009, 80, 061908.

[27] J. Chin, P. V. Coveney, Proc. R. Soc. A 2006, 462, 3575.

[28] K. Michielsen, D. G. Stavenga, J. R. Soc. Interface 2008, 5, 85.

[29] L. Poladian, S. Wickham, K. Lee, M. C. J. Large, J. R. Soc. Interface 2009, 6, S233.

[30] M. Saba, B. D. Wilts, J. Hielscher, G. E. Schröder-Turk, Materials Today: Proceedings 2014, $1,193$.

[31] W. Man, M. Megens, P. J. Steinhardt, P. M. Chaikin, Nature (London, U. K.) 2005, 436, 993.

[32] C. Pouya, P. Vukusic, Interface Focus 2012, 2, 645.

[33] P. Vukusic, J. R. Sambles, Proc. SPIE 2001, 4438, 85.

[34] P. Vukusic, J. R. Sambles, Nature (London, U. K.) 2003, 424, 852.

[35] B. D. Wilts, K. Michielsen, H. De Raedt, D. G. Stavenga, Interface Focus 2012, 2, 681. 


\section{WILEY-VCH}

[36] V. Saranathan, C. O. Osuji, S. G. J. Mochrie, H. Noh, S. Narayanan, A. Sandy, E. R. Dufresne, R. O. Prum, Proc. Natl. Acad. Sci. U. S. A. 2010, 107, 11676.

[37] S. Yoshioka, B. Matsuhana, H. Fujita, Materials Today: Proceedings 2014, 1, 186.

[38] S. Yoshioka, H. Fujita, S. Kinoshita, B. Matsuhana, J. R. Soc., Interface 2014, 11, 20131029.

[39] C. Pouya, Ph.D. Thesis, University of Exeter, UK, June, 2012.

[40] M. R. J. Scherer, Double-Gyroid-Structured Functional Materials: Synthesis and Applications, Springer Science \& Business, Springer International Publishing Switzerland 2013.

[41] K. Michielsen, J. S. Kole, Phys. Rev. B: Condens. Matter Mater. Phys. 2003, 68, 115107.

[42] P. J. F. Gandy, J. Klinowski, Chem. Phys. Lett. 2000 321, 363.

[43] M. Wohlgemuth, N. Yufa, J. Hoffman, E. L. Thomas, Macromolecules (Washington, DC, U. S.) 2001, 34, 6083 .

[44] H. G. von Schnering, R. Nesper, Z. Phys. B: Condens. Matter 1991, 83, 407.

[45] Y. Grin, R. Nesper, Z. Kristallogr. - Cryst. Mater. 2011, 226, 692.

[46] A. M. Nicolson, G. F. Ross, IEEE Instrumentation \& Measurement Magazine 1970, 19, 377.

[47] W. B. Weir, Proc. IEEE 1974, 62, 33. 


\section{WILEY-VCH}

A
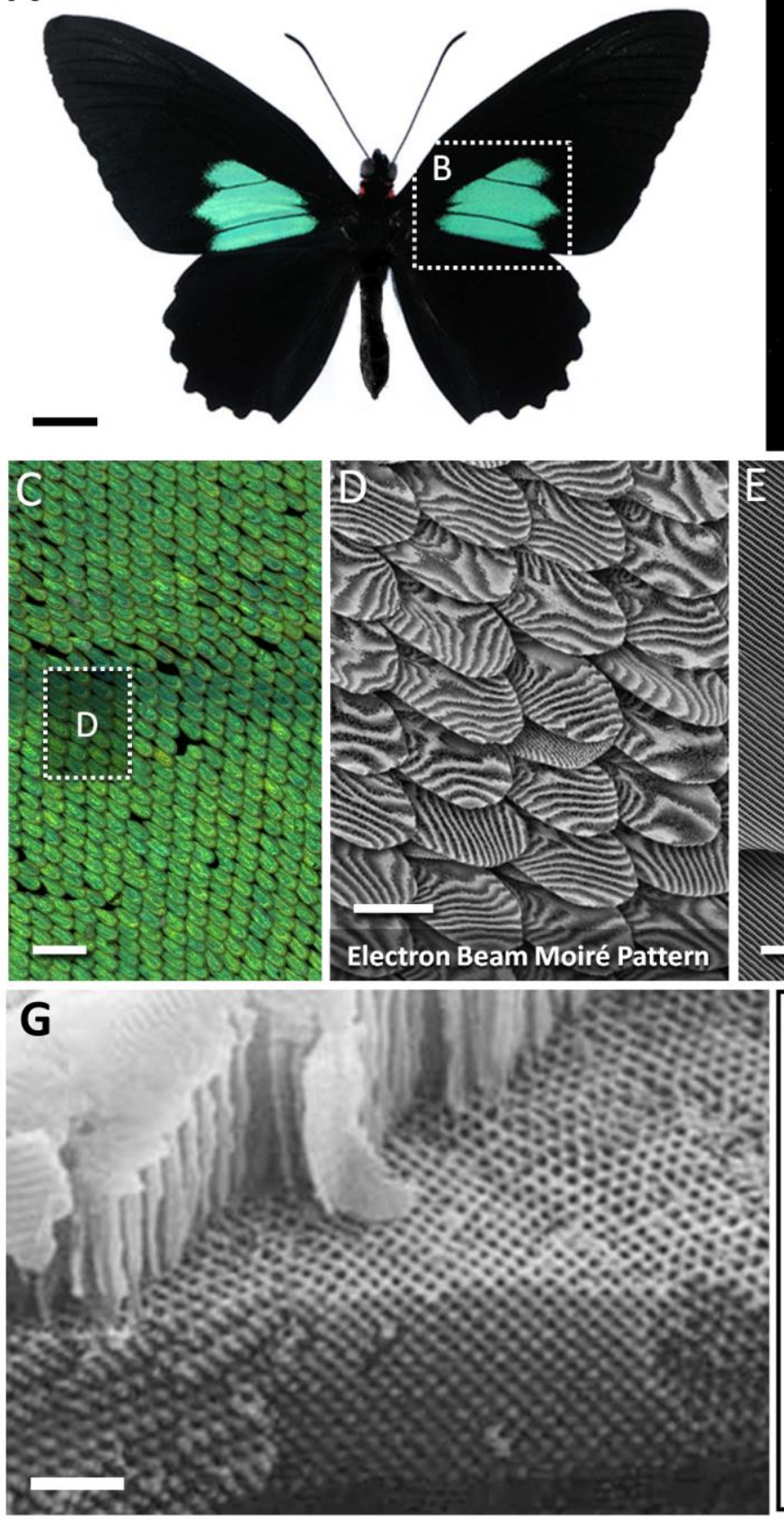
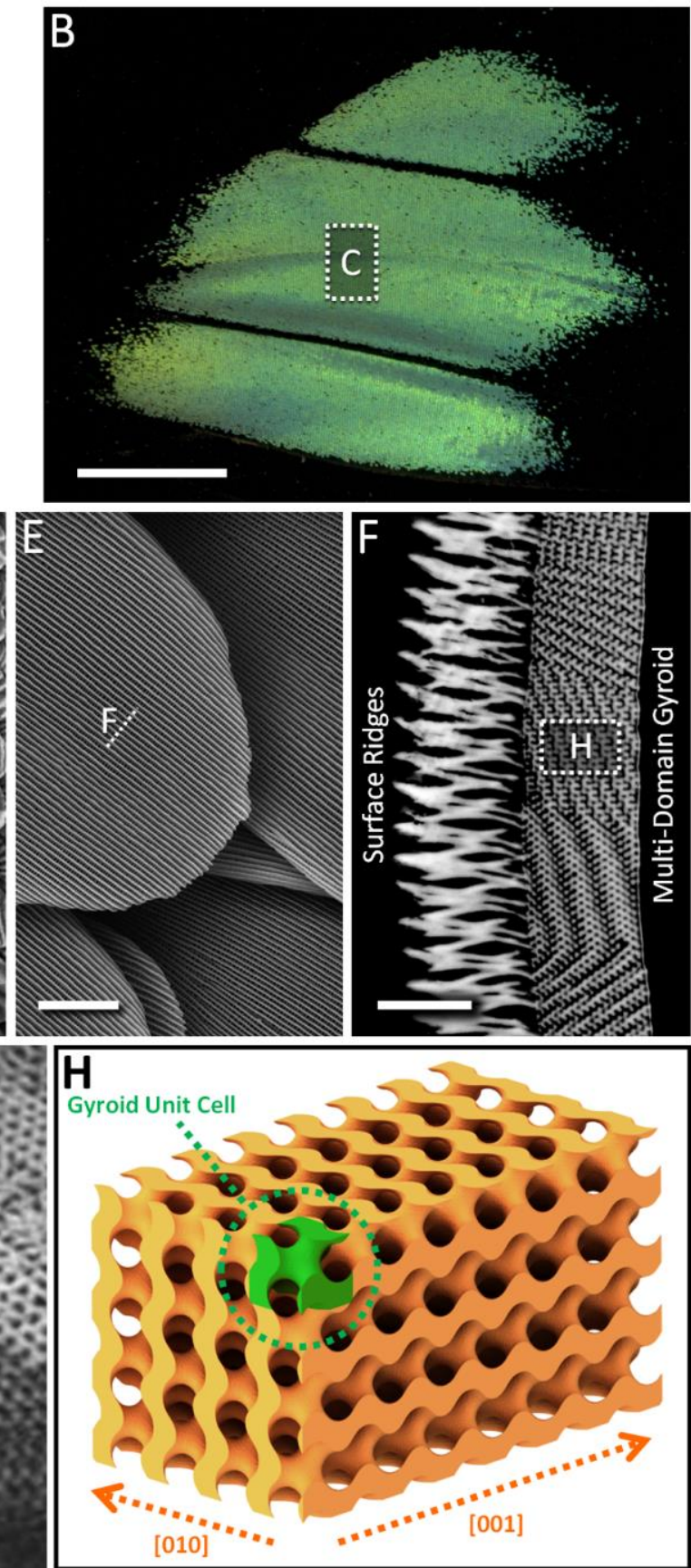

\section{Figure 1.}

Mechanistic origins of structural color in the neotropical butterfly, Parides sesostris. Located on the wings of P. sesostris (A) are striking metallic green patches (B) and high-resolution structural characterizations of their constituent scales (C-F) reveals a complex composite architecture consisting of surface ridges $(\mathrm{E})$ and an underlying photonically active multidomain gyroid $(\mathrm{F}) .^{[33,34]}(\mathrm{D})$ shows an electron beam Moiré pattern, generated by matching the e-beam scanning periodicity with the structural periodicity of the wing scales. $(G)^{[33,34]}$ shows a the 3D structure present within P.sesostris wing scales, which can be graphically represented by the photonic gyroid structure $(\mathrm{H})$ with an individual unit cell highlighted in green. For reference, each scale measures ca. $150 \mu \mathrm{m}$ in length. Scale bars: (A) $1 \mathrm{~cm}$, (B) 5 mm, (C) $200 \mu \mathrm{m}$, (D) $50 \mu \mathrm{m}$, (E) $10 \mu \mathrm{m}$, (F) $4 \mu \mathrm{m}$, (G) $2.5 \mu \mathrm{m}$. 


\section{WILEY-VCH}

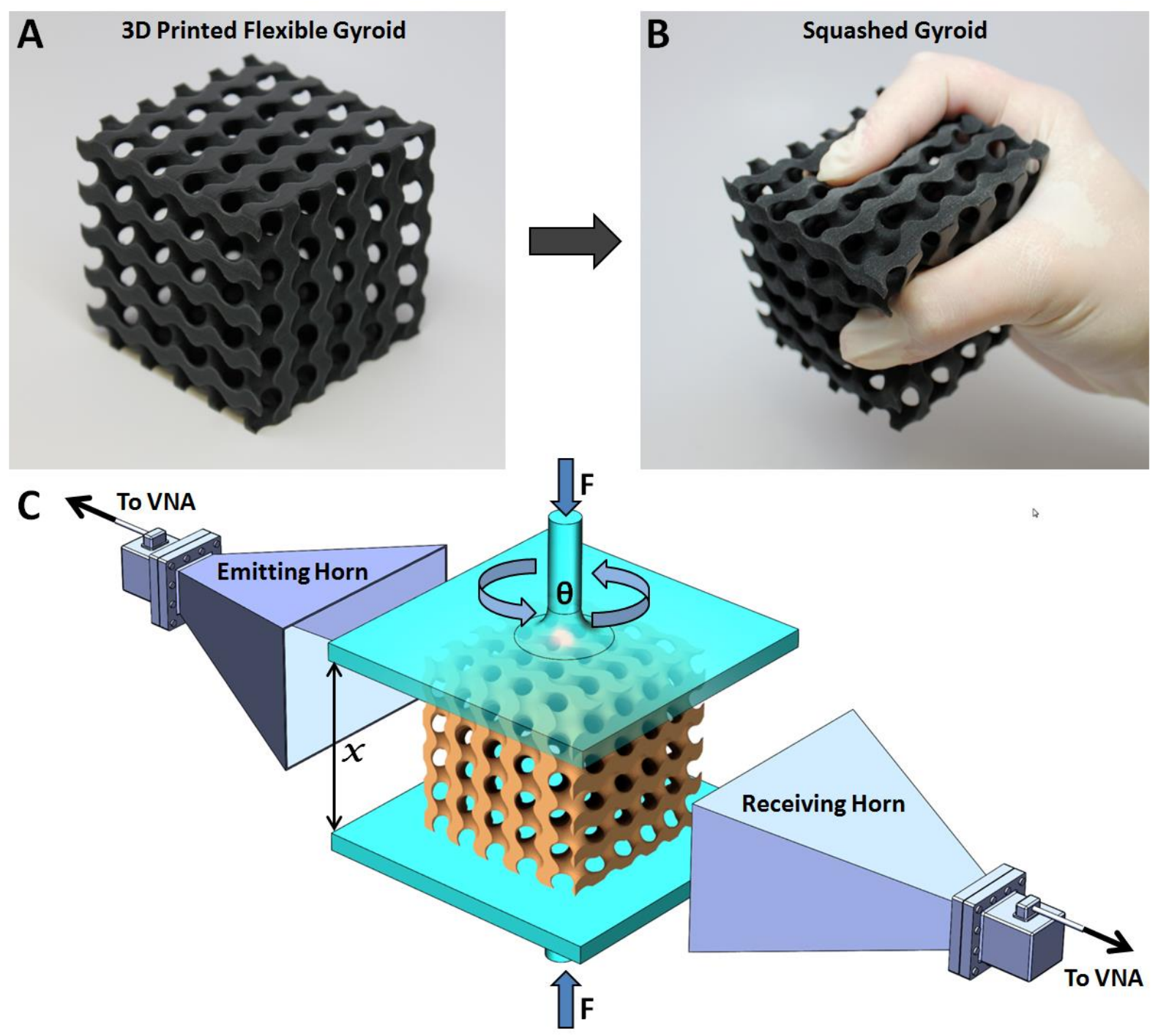

Figure 2.

Fabrication and characterization of the gyroid photonic crystal. High resolution additive manufacturing was used to generate a compressible gyroid (A, B) from a rubber-like polymer (permittivity of $\varepsilon=2.85+0.15 i$ ). (C) illustrates a schematic diagram of the microwave transmission experimental set-up, showing the relative positions of the broadband horns, the gyroid photonic crystal sample, and the compression device. 


\section{WILEY-VCH}

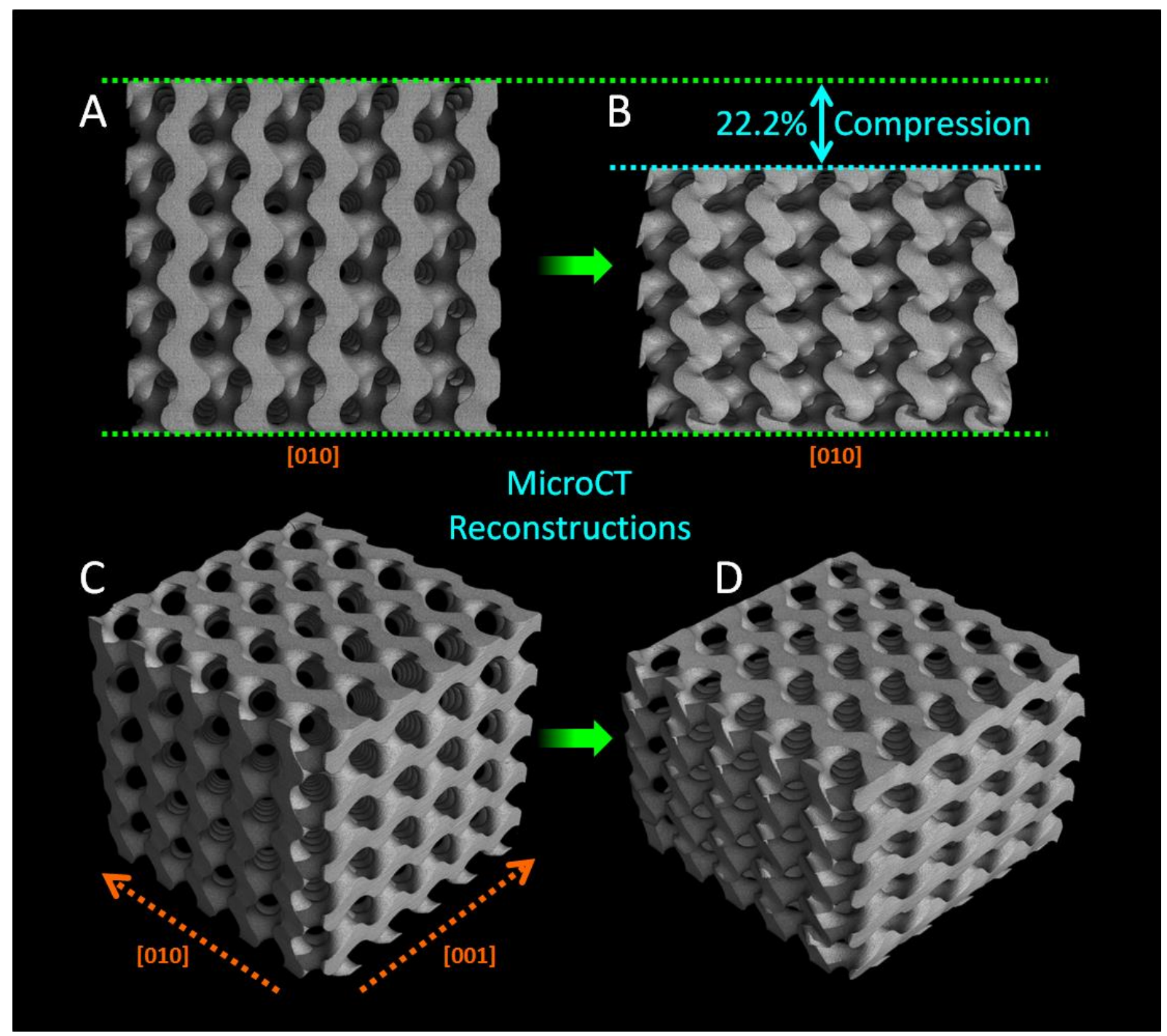

\section{Figure 3.}

MicroCT reconstructions of the compressible gyroid. X-ray computed micro-tomographic reconstructions of the 3D-printed gyroid in its uncompressed state and when compressed by $22.2 \%$ of its original size, clearly showing the reduction of volume fraction upon loading and the corresponding lateral expansion of the structure. 


\section{WILEY-VCH}

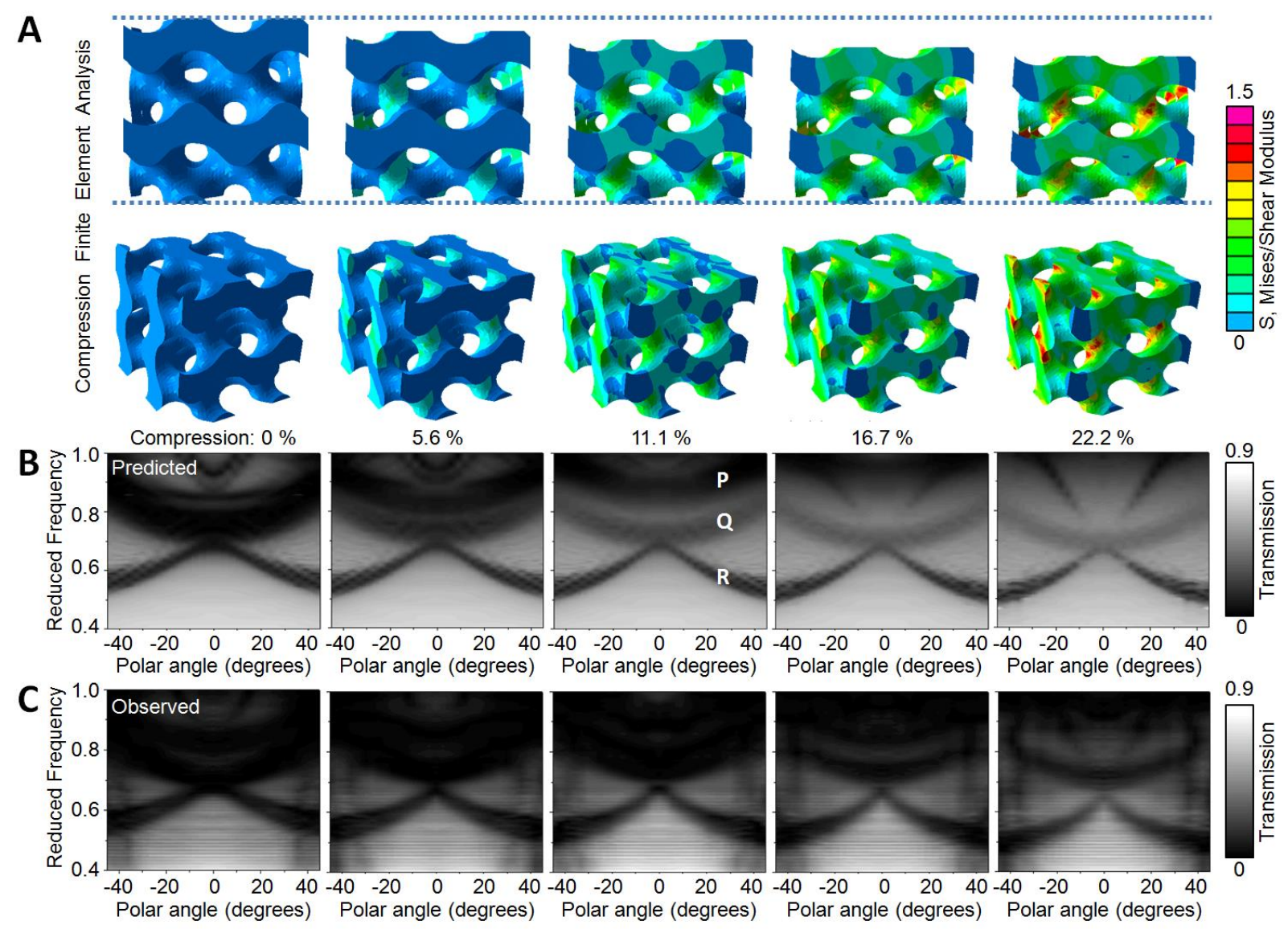

Figure 4.

Finite element compression simulations (A) of the gyroid and its corresponding theoretical (B) and experimental photonic responses (C). FEA simulations of the flexible gyroid (A) reveal a relatively uniform strain field through the entire experimental loading regime. The results shown here are extracted from a $6 \times 6 \times 6$ unit cells simulation, and show the internal deformation, which is not affected by boundaries. Theoretical (B) and experimental (C) transmitted field magnitude data showing the resonant electromagnetic features associated with the gyroid photonic crystal structure with an initial material volume fraction of $40 \%$ and lattice constant of $9 \mathrm{~mm}$ using transverse magnetic linearly polarized incident radiation. ${ }^{\text {[39] }}$ The structure was compressed by $0 \%, 5.6 \%, 11.1 \% 16.7 \%$, and $22.2 \%$, from left to right in (B) and (C), across the [100] direction. A reduced frequency regime was employed, normalized by the initial lattice constant value of $\mathrm{a}=9 \mathrm{~mm}$ and $c$, the speed of light in free space. The sample was rotated over a polar angle range of $-45^{\circ} \leq \theta \leq 45^{\circ}$ at an azimuthal angle of $\varphi=0^{\circ}$ for each compressed state. ${ }^{[39]}$ The labels ' $P$ ', ' $Q$ ', and ' $R$ ' (shown only in the central graph of Figure 4B) refer to individual dispersion features that originate from resonances in the $[\overline{1} 0 \overline{1}],[00 \overline{1}]$, and $[0 \overline{1} \overline{1}]$ directions, respectively, though the gyroid. 